\title{
Some topics in non-singular rings
}

\section{M.W. Evans}

A ring $R$ is right non-singular if and only if $Q_{R}$, the maximal quotient ring of $R$, is von Neumann regular. A ring $R$ is said to be a right P.P. ring if every principal right ideal of $R$ is projective. A right P.P. ring is right non-singular.

Chapter 1 is expository. In the first three sections of Chapter 2, the condition that $Q_{R}$ is left torsion-free in the sense of Hattori ([2]) is considered. When $R$ is commutative and non-singular it is shown that this condition is equivalent to the left flat epimorphic hull of $R$ being a regular ring. The fourth section of the chapter is an investigation of the left flat epimorphic hull of a not necessarily commutative non-singular ring and some sufficient conditions are found for it to be regular. Ideals of a comatative non-singular ring $R$ which are contractions of ideals in the maximal quotient ring of $R$ are considered in Section 5 .

Chapter 3 is principally concerned with right P.P. rings and several characterisations of these rings are collected. It is shown that a ring $R$ is right P.P. If and only if every principal right ideal of $R$ is flat and there exists a ring monomorphism $\phi: R \rightarrow S$, where $S$ is a regular ring which is left torsion-free, in the sense of Hattori, when considered as an $R$-module.

A right $R$-module $A_{R}$ is said to be right projectively torsion-free (P.T.F.) if for every $a \in A$ there exist $s_{1}, s_{2}, \ldots, s_{n} \in R$ and $a_{1}, a_{2}, \ldots, a_{n} \in A$ such that $a=\sum_{j=1}^{n} a_{j} s$ and if $a x=0$ for some

Received 25 September 1973. Thesis submitted to Monash University, February 1973. Degree approved, August 1973. Supervisor: Professor P.D. Finch. 
$x \in R$, then $s_{j} x=0$ for all $l \leq j \leq n$. It is shown that these form a hereditary torsion-free class of $\bmod R$ if and only if each right complement ideal of $R$ is generated by an idempotent and $R$ is right nonsingular. Characterisations of right P.P. rings and Baer rings are also presented through these modules.

Chapter 4 is a discussion of right semi-hereditary rings. Chapter 5 is concerned with the epimorphic hull ([3]) of a commutative non-singular ring and a simple construction is given for it. It is also shown that the epimorphic hull of a commutative non-singular ring satisfies a certain universal property.

\section{References}

[1] M.W. Evans, "On commutative P.P. rings", Pacific J. Math. 41 (1972), 687-697.

[2] Akira Hattori, "A foundation of torsion theory for modules over general rings", Nagoya Math. J. 17 (1960), 147-158.

[3] Hans Heiner Storrer, "Epimorphismen von kommutativen Ringen", Comment. Math. Helv. 43 (1968), 378-401. 